\title{
BMJ Open Frequency and format of clinical trial results dissemination to patients: a survey of authors of trials indexed in PubMed
}

\author{
Sara Schroter (D) , ${ }^{1}$ Amy Price (D) , ${ }^{1}$ Mario Malički, ${ }^{2}$ Tessa Richards, ${ }^{1}$ \\ Mike Clarke (1) ${ }^{3}$
}

To cite: Schroter S, Price A, Malički M, et al. Frequency and format of clinical trial results dissemination to patients: a survey of authors of trials indexed in PubMed. BMJ Open 2019;9:e032701. doi:10.1136/ bmjopen-2019-032701

- Prepublication history and additional material for this paper are available online. To view these files, please visit the journal online (http://dx.doi. org/10.1136/bmjopen-2019032701).

Received 01 July 2019 Revised 16 September 2019 Accepted 30 September 2019

Check for updates

(C) Author(s) (or their employer(s)) 2019. Re-use permitted under CC BY-NC. No commercial re-use. See rights and permissions. Published by BMJ.

${ }^{1}$ The BMJ, London, UK ${ }^{2}$ Department of Research in Biomedicine and Health, University of Split School of Medicine, Split, Croatia

${ }^{3}$ Northern Ireland Methodology Hub, Queen's University Belfast, Belfast, UK

Correspondence to

Dr Sara Schroter;

sschroter@bmj.com

\section{ABSTRACT}

Objective Dissemination of research findings is central to research integrity and promoting discussion of new knowledge and its potential for translation into practice and policy. We investigated the frequency and format of dissemination to trial participants and patient groups. Design Survey of authors of clinical trials indexed in PubMed in 2014-2015.

Results Questionnaire emailed to 19321 authors; 3127 responses received (16\%). Of these 3127 trials, 2690 had human participants and 1818 enrolled individual patients. Among the 1818, 498 authors (27\%) reported having disseminated results to participants, $238(13 \%)$ planned to do so, 600 (33\%) did not plan to, $176(10 \%)$ were unsure and $306(17 \%)$ indicated 'other' or did not answer. Of the 498 authors who had disseminated, 198 (40\%) shared academic reports, $252(51 \%)$ shared lay reports, $111(22 \%)$ shared both and $164(33 \%)$ provided individualised study results. Of the 1818 trials, 577 authors $(32 \%)$ shared/planned to share results with patients outside their trial by direct contact with charities/patient groups, 401 (22\%) via patient communities, 845 (46\%) via presentations at conferences with patient representation, $494(27 \%)$ via mainstream media and $708(39 \%)$ by online lay summaries. Relatively few of the 1818 authors reported dissemination was suggested by institutional bodies: 314 $(17 \%)$ of funders reportedly suggested dissemination to trial participants, $252(14 \%)$ to patient groups; 333 $(18 \%)$ of ethical review boards reportedly suggested dissemination to trial participants, $148(8 \%)$ to patient groups. Authors described many barriers to dissemination. Conclusion Fewer than half the respondents had disseminated to participants (or planned to) and only half of those who had disseminated shared lay reports. Motivation to disseminate results to participants appears to arise within research teams rather than being incentivised by institutional bodies. Multiple factors need to be considered and various steps taken to facilitate wide dissemination of research to participants.

\section{INTRODUCTION}

Dissemination of research findings is central to research integrity and supports the translation of new knowledge into policy and practice. Dissemination is the process by which
Strengths and limitations of this study

- Largest survey of current dissemination practices of authors of biomedical research.

- Describes frequency and format of what was disseminated

- A survey can only report what authors said they did, not what they actually did in practice.

findings are communicated to target audiences, including policy makers, academics, health professionals, patients, the public, and research funders. Dissemination is considered to be a shared responsibility between funders and researchers but there is variation in expectations and practice. ${ }^{12}$ Traditionally, dissemination has targeted health professionals, policy makers and academics through journal publications and presentations at conferences. ${ }^{3}$ Researchers are encouraged and motivated to publish and disseminate to their peers to advance their careers ${ }^{4}$ and to satisfy processes such as the Research Excellence Framework in the UK (www.ref. ac.uk). There are no such strong motives to drive dissemination to patient participants or communities of patients to whom the findings might be relevant.

Dissemination to participants has attracted debate and many agree it is an ethical imperative and essential marker of respect to people who participate in research studies, which often place a high burden on their time and energy. ${ }^{5-8}$ Furthermore, the Declaration of Helsinki, the set of ethical principles regarding human experimentation developed by the World Medical Association states that "all medical research subjects should be given the option of being informed about the general outcome and results of the study". ${ }^{9}$ Regulatory authorities have recently stipulated that results of clinical trials must be published in 
open-access databases ${ }^{10}$ and recommendations for sharing individual research data have also been made ${ }^{11}$ although these have different ethical and practical implications to the dissemination of a study's aggregated results. ${ }^{12} 13$ Some funders, including the Patient-Centered Outcomes Research Institute in the USA and the National Institute for Health Research (NIHR) in the UK, ask researchers to share results with study participants, but there is debate on what dissemination to patients means and how best to do it. ${ }^{14-16}$

The frequency with which results are shared with participants has been investigated ${ }^{617-20}$ but routine practice in this respect is not known. Nor is whether results are shared in an easy to read format, despite participants' interest to read them and researchers' willingness to share them. ${ }^{12122}$ This paper reports a large survey of researchers from a wide range of clinical specialties about a specific clinical trial they had recently published, to determine the frequency and format of dissemination to trial participants and wider patient communities, and to explore barriers to doing so.

\section{METHODS}

\section{Questionnaire development and administration}

\section{Development and pretesting}

The survey (online supplementary appendix 1 ) was developed by the authors, piloted with 10 researchers to check for ambiguous questions and that the skip format was appropriate, and revised as necessary.

\section{Patient and public involvement}

Two members of the research team are patients living with long-term medical conditions. All members of the research team and one of The BMJ's former patient editors helped develop the questionnaire, which was reviewed by two members of The BMJ's international patient advisory panel; who also commented on the draft manuscript.

\section{Survey administration}

Identified authors (see below) were sent an invitation to complete the survey by email on 24 January 2016 using SurveyMonkey. The email offered entry into a prize draw for an Amazon voucher. Reminder emails were sent to non-respondents two and 4 weeks following the initial invitation. Completion of the survey was taken to indicate consent to participate. Authors could choose not to take part and were notified that they were free to stop and withdraw at any time.

\section{Sampling and search strategy}

The targeted sample was authors of clinical trials published in 2014 or 2015 in journals indexed in PubMed. While issues of dissemination to participants are potentially of importance to all research involving patients, we focused on clinical trials because of the way they are indexed and coded (and therefore accessible) and because they are a major element in the evidence base for healthcare. We identified them using two searches and removed duplicate titles (as initially we planned to focus on just 1 year): (clinical trial[Publication Type] AND ('2014'[PDAT]: '2014'[PDAT])) NOT 'clinical trials as topic' [MeSH Terms] and (clinical trial[Publication Type] AND ('2015'[PDAT]: '2015'[PDAT])) NOT 'clinical trials as topic' [MeSH Terms]. We used a Perl script to extract the email address of the first named author from the affiliation section of the record, along with the following variables from the PubMed XML: first author's first name, first author's surname, journal name, publication date and article title. We excluded email addresses if there was no named author.

One person was invited to participate per trial report. To avoid sending more than one invitation to authors with two or more eligible trials, we removed trials with duplicate email addresses. We also excluded authors who had previously asked not to be contacted by $B M J$ for marketing and other reasons.

\section{Eligibility criteria and screening question}

We included authors of published protocols because protocols are not categorised/indexed in PubMed in a way that would allow them to be easily identified. Additionally, trials for which the protocol was published in 2014 or 2015 might have been completed by the time of the survey and authors were free to respond in relation to the completed study. If they had not yet disseminated, authors were asked if they had plans to disseminate so they could also respond about a protocol in this way.

Only reports of clinical trials in humans were included in the analysis. We included a screening question at the start of the survey to exclude reports of laboratory or animal studies, preclinical trials without patients, studies where there were no study participants, and studies that were not clinical trials.

\section{Data analysis}

Data are presented as number and percent of the total number of authors of eligible trials with individual patients recruited. Open ended answers were grouped into themes by SS and verified by AP.

\section{RESULTS \\ Sample}

The PubMed search identified 26185 clinical trial reports, but we subsequently excluded 5798 reports (see figure 1). We used SurveyMonkey to send emails to authors of 20387 reports, but 1066 resulted in delivery failures. 3127/19 $321(16 \%)$ responded to the survey and a further 88 emailed to disqualify themselves (stating their publication was: not a clinical trial $n=48$, a protocol with no results available $n=20$, secondary analyses $n=15$, a methodological paper $n=2$, an animal study $n=2$, a laboratory study $\mathrm{n}=1$ ). Of the 3127 who responded to the first screening question, 437 were disqualified from taking part as they stated their publication was not a clinical trial 


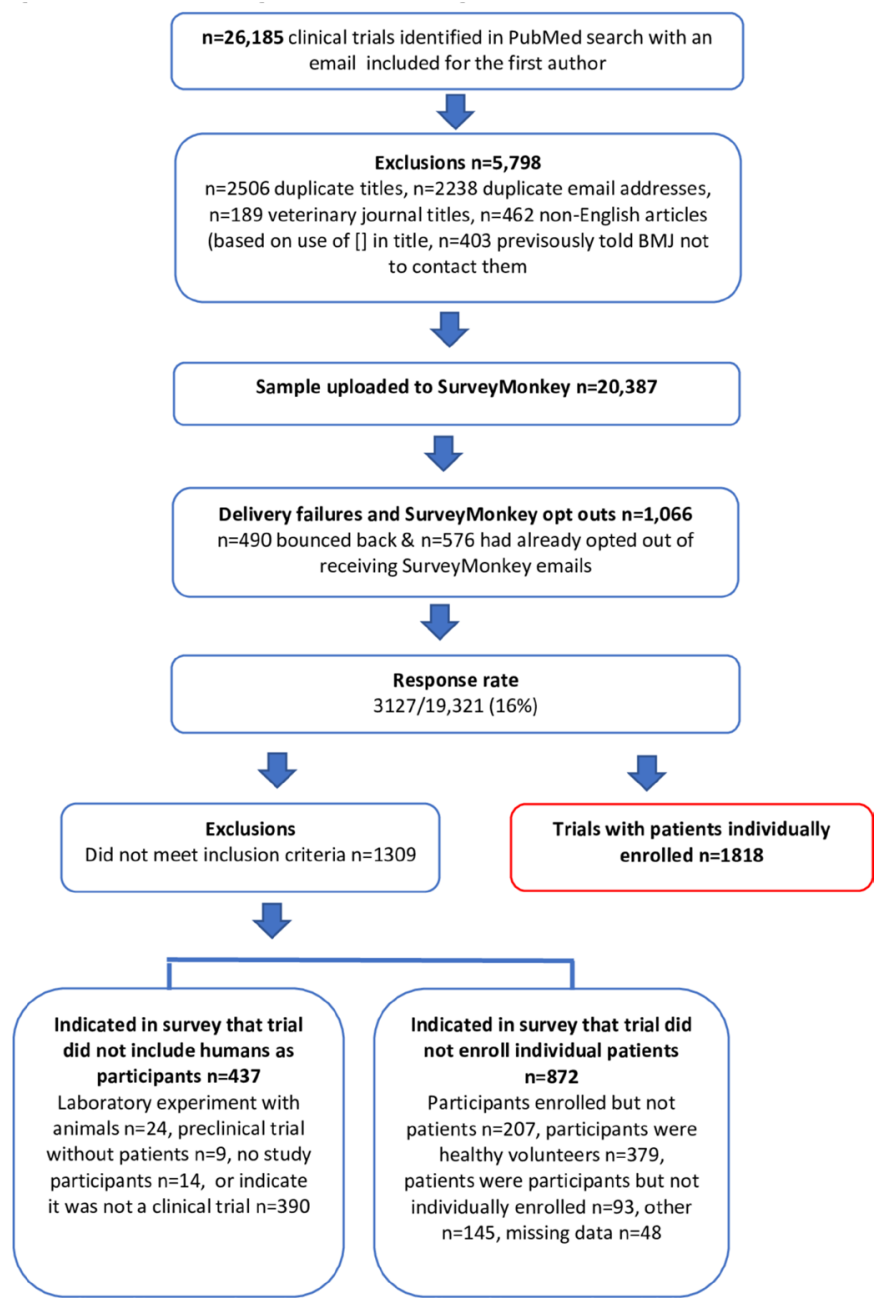

Figure 1 Flowchart indicating response rate and eligible trials.

$(\mathrm{n}=390)$ or that it was a trial without human participants $(\mathrm{n}=47)$. Of the remaining 2690 trials, 1818 with patients individually enrolled are the focus of this paper.

\section{Respondent and trial characteristics}

The 1818 respondents were working in 71 different countries, mostly in universities $(50 \%)$ or hospital or health institutions $(27 \%)$ and had been active researchers for a broad range of years (table 1 ). The trials were published in over 600 different journals and 345 (19\%) were reported as having some funding from industry, 905 $(50 \%)$ had some non-industry funding, and 538 (30\%) had no external funding.

\section{Dissemination to trial participants}

$498(27 \%)$ of the 1818 authors reported that they had already disseminated results to participants and another $238(13 \%)$ planned to do so, $600(33 \%)$ did not plan to, $176(10 \%)$ were unsure, and $306(17 \%)$ responded with 'other' or did not answer.

As we received a low response rate for the survey and those who did not respond may have been less likely to have disseminated we report some sensitivity analysis: if non-responders were $20 \%$ less likely to have disseminated
Table 1 Respondent characteristics for 1818 trials where patients were individually enrolled

\begin{tabular}{lc}
\hline $\begin{array}{l}\text { Respondents' work and clinical trial } \\
\text { experience }\end{array}$ & $\mathbf{n ~ ( \% )}$ \\
\hline $\begin{array}{l}\text { Type of work institution } \\
\text { University }\end{array}$ & $903(50)$ \\
\hline Private research centre & $53(3)$ \\
\hline Public research centre & $57(3)$ \\
\hline Hospital or health institution & $492(27)$ \\
\hline Industry & $22(1)$ \\
\hline Other & $36(2)$ \\
\hline Number of years as an active researcher & \\
\hline $1-5$ & $268(15)$ \\
\hline $6-10$ & $432(24)$ \\
\hline $11-20$ & $489(27)$ \\
\hline $21-30$ & $251(14)$ \\
\hline More than 30 & $121(7)$ \\
\hline Proportion of clinical trials with patients that respondents \\
had been involved in where results were disseminated to \\
participants
\end{tabular}

Percentages do not sum to $100 \%$ due to missing data.

(or have plans to disseminate) than responders, then the overall proportion who have disseminated (or have plans to) would be $21 \%(4137 / 19321)$ and if non-responders were $40 \%$ less likely it would be $5 \%(898 / 19321)$.

For $1225(67 \%)$ of the 1818 trials, at least one of the author team had access to participant identifiable data. Of the 600 respondents who indicated that there were no plans to disseminate, $418(70 \%)$ confirmed that authors had access to the identifiable data.

Of the 498 authors who had disseminated results to participants, 198 (40\%) shared documents or presentations they had prepared for an academic/clinical audience, $252(51 \%)$ shared documents or presentations prepared specifically for lay readers, $111(22 \%)$ shared both academic documents and materials prepared specifically for lay readers, and $164(33 \%)$ provided individual study results (table 2). When trial participants were offered lay documents or presentations, these were most frequently $(34 \%, \mathrm{n}=170)$ sent directly to them; $10 \%$ $(n=48)$ of authors made the results available on a website and $16 \%(\mathrm{n}=82)$ invited participants to a meeting. Where documents or presentations written for an academic audience were shared, the journal publication was most frequently shared $(28 \%, \mathrm{n}=139)$, followed by an academic 
Table 2 Type of information offered to the participants in trials for which authors had already disseminated or planned to disseminate results

\begin{tabular}{|c|c|c|c|}
\hline \multirow[b]{2}{*}{ Type of information } & \multicolumn{3}{|l|}{$\mathbf{N}(\%)$ of trials } \\
\hline & $\begin{array}{l}\text { Already } \\
\text { disseminated to } \\
\text { participants, } n=498\end{array}$ & $\begin{array}{l}\text { Plan to disseminate } \\
\text { to participants, } \\
\mathrm{n}=238\end{array}$ & $\begin{array}{l}\text { Already } \\
\text { disseminated } \\
\text { or plan to } \\
\text { combined, } \\
\mathrm{n}=736\end{array}$ \\
\hline $\begin{array}{l}\text { Documents or presentations written for an academic/clinical } \\
\text { audience }\end{array}$ & $198(40)$ & $105(44)$ & $303(41)$ \\
\hline Journal publication(s) & $139(28)$ & $67(28)$ & $206(28)$ \\
\hline Academic summary & $74(15)$ & $47(20)$ & $121(16)$ \\
\hline Documents or presentations prepared specifically for lay readers & $252(51)$ & $114(48)$ & $366(50)$ \\
\hline Participants sent a lay summary & $170(34)$ & $82(35)$ & $252(34)$ \\
\hline $\begin{array}{l}\text { Individualised study results, such as outcomes, scores or } \\
\text { analysed data }\end{array}$ & $164(33)$ & $49(21)$ & $213(29)$ \\
\hline
\end{tabular}

Authors could indicate multiple options.

summary $(15 \%, \mathrm{n}=74)$ or the full study report $(11 \%$, $\mathrm{n}=56)$.

$906(50 \%)$ of the 1818 authors reported that trial participants had been asked if they wanted to receive the study results. 571 of these 906 (63\%) authors had already disseminated trial results to participants or planned to do so, but $142(16 \%)$ had no plans to disseminate to the participants.

\section{Perceived benefits of dissemination of results to patients}

Respondents' comments on the benefits of disseminating results to patients included supporting the spread of knowledge in the patient community, increased accountability for researchers, and an opportunity to empower patients. The potential to motivate people to participate in future research studies was noted by many and some suggested dissemination might encourage patients to consider interventions which could lead to better outcomes for them. It was also suggested that it might improve the doctor-patient relationship through building confidence and trust. Respondents further suggested that the impact of dissemination could be extended by giving patients the opportunity to share results within their own communities; there were mixed views on whether dissemination should be mandatory.

Barriers or challenges to dissemination to patient participants All participants were asked to describe barriers or challenges to dissemination and we grouped these into seven key themes (box 1 contains illustrative quotes). It is important to note, however, that some authors reported that there were no barriers to dissemination and described successful approaches.

Researchers' perceptions of what interests patients and what they can understand

Some researchers said they did not think patients would be interested in receiving study results; others assumed a failure to ask for them represented a lack of interest in getting them. Many were concerned that patients would lack the ability to understand the results and their implications.

\section{Difficulties in reaching patients}

Respondents described potential problems such as what to do about dissemination if participants may have died, and how to reach patients who don't have access to the internet, are illiterate, critically ill, cognitively impaired, minors, or in other vulnerable groups. Dissemination was also seen as challenging if a long time had elapsed between conducting the study and its publication. Additional difficulties cited included not being able to give personal feedback; having to disseminate in different languages; difficulty in writing lay summaries; and only having access to anonymised datasets. Some felt restricted by data protection rules and some were not confident about how and when they could contact trial participants in the absence of relevant guidelines.

Lack of early planning and support

Some researchers reported that they did not have ethical approval or patient consent for dissemination, plans to 
Box 1 Illustrative quotes describing barriers and challenges to disseminating to trial participants

Researchers' perceptions of what interests patients and what they can understand

"The results has scientific and medical interest, but they are not interesting nor directly useful for patients". (17347)

"Basically the patients are operated and doing well and therefore they are not very interested any longer-they care about themselves and not others". (19292)

"Study participants are interested only in their health recovery and usually don't ask about study results". (9096)

"Patients should be given info if they request results otherwise this should not become mandatory - it would become another unnecessary task mandated to researchers". (2772)

"Small part of abdominosurgical procedure, difficult to understand for patient, no further consequence". (14807)

\section{Difficulties in reaching patients}

"You cannot give information to participants that is not interpreted. As many will fall well below norms or are clearly not doing well, dissemination without the opportunity to intervene is unethical .... so I will not do it". (6117)

"One difficulty is the gap in time between when the study is completed and the results are available. The study is closed for IRB purposes. Adding another communication to patients will require additional work at all levels, from the sponsor to the investigator to the IRB". (5247)

\section{Lack of early planning and support}

"We did not get IRB approval at the time of the study to contact participants following their participation in the study. Also, since the study is now closed with the IRB, we are not to have contact with the participants". (7460)

"IRBS and regulatory bodies in my country do not put emphasis or a sense of obligation to ensure that study results reach the participants. It is not mandatory at the time of approval of a study and little or no follow-up is done". (8580)

"It needs to be required by EMA and FDA and all ethics committees and built into the protocols. It also needs to be funded as part of the study. Currently, in study budgets, there is not a line item to address the process". (2126)

\section{Lack of incentives and cultural expectations}

"To be blunt, I am an academic researcher and there is no incentive for me to do it, other than that it is the right thing to do. There is no pressure from funders or my department, and it doesn't factor in to my promotion so it feels like something extra I need to make time for". (10880)

"Gets lost in the shuffle of so many other priorities and it is not mandated. But I would imagine participants would be interested in knowing the results". (9025)

"There is also the issue of what academic credit one gets. Eg people will spend time writing papers after finishing on a project but they will get no acknowledgement for spending time preparing lay summaries and actively disseminating to patients. There needs to be trial manager/research associate time built into grant funding for the post-trial dissemination. There also needs to be a shift so that dissemination to patients and public carries more weight than it currently does. For example whilst I am passionate about this and feel it is more important in many ways than writing a journal article I have never been set an objective to go to a patient group to discuss the trials but have been set objectives about writing articles". (5750)

Continued

\section{Box 1 Continued}

“... for annual review and promotion, disseminating results to patients does not count whereas publishing in a journal or presenting at a professional meeting does count". (7555)

"Just not a part of the research culture I work in. Would be easy enough to do ....". (8490)

"Because it is not expected to disseminate results to patients involved in the study". (2094)

"We weren't trained as researchers to think in this way ... It is not part of the grant getting, research publishing environment we work in". (11511)

\section{Type of results}

"The results have no bearing on the care/management of the patients who participated". (9612)

"The trial results would concern the participants about their future. However, they could not change their own risk in the future". (7996)

"If the trial has a negative outcome maybe the patients feel that they waisted their time participating in the trial". (5794)

"The main barrier is to determine whether the results are meaningful, reproducible and helpful to the patients involved. If they are not, they should not be disseminated to patients". (10704)

\section{Uncertainty on which patient groups to share study results with}

"... there are patients with chronic diseases and with acute diseases like trauma, sepsis, ARDS, cardiac arrest; and these do not have patients groups". (8212)

"There are no patient organisation representing critically ill patients ... and thus no obvious group of patients outside of the study to approach". (3916)

\section{Researcher-specific reasons for non-dissemination}

"It's not standard practice here or anything I've seen done by coauthors or mentors. Until I saw this survey it honestly never occurred to me to offer this". (19036)

"Didn't think of it at the time. I absolutely will in the future. Time and cost is secondary". (10267)

"Participants can access the result with published article". (3743)

"The results are published and so accessible for anyone that is interested". (10846)

do so were not included in the study protocol and results were not offered to participants at the outset. Earmarked financial support for dissemination was unusual, and where it was available, it was often not accessible by the time the study was published. Researchers also stated that funders did not require or mandate dissemination and that it would provide an additional burden to the research team.

\section{Lack of incentives and cultural expectations}

Respondents described the lack of expectation, academic recognition or reward for disseminating to participants and patient groups compared with disseminating to researchers and clinical audiences.

Type of results

Study-specific reasons included concern over misinterpretation of unclear, inconclusive or discouraging study results; negative results; preliminary results; and complex 
Table 3 Dissemination of results to patients outside of those who took part in the trial $(n=1818)$

\begin{tabular}{|c|c|c|c|c|}
\hline \multirow[b]{2}{*}{ Dissemination method } & \multicolumn{4}{|c|}{$\mathbf{N}(\%)$ of trials } \\
\hline & $\begin{array}{l}\text { Already } \\
\text { shared }\end{array}$ & Plan to share & $\begin{array}{l}\text { Nether } \\
\text { shared nor } \\
\text { plan to }\end{array}$ & $\begin{array}{l}\text { Don't } \\
\text { know }\end{array}$ \\
\hline By direct contact with relevant patient charities or patient groups & $379(21)$ & $198(11)$ & $692(38)$ & $199(11)$ \\
\hline Via informal patient communities (eg, shared on social media) & $248(14)$ & $153(8)$ & $780(43)$ & $209(12)$ \\
\hline Via presentations at conferences where patients are represented & $650(36)$ & $195(11)$ & $492(27)$ & $153(8)$ \\
\hline $\begin{array}{l}\text { By publishing lay summaries on the trial website or somewhere } \\
\text { publicly accessible }\end{array}$ & $432(24)$ & $276(15)$ & $540(30)$ & $206(11)$ \\
\hline
\end{tabular}

Percentages do not sum to $100 \%$ due to missing data.

or technical results (eg, a comparison of surgical techniques). Some felt that it was not necessary to disseminate results of all studies, particularly when findings would not change management, or would be of no benefit to individual participants; or where knowing the results might cause distress.

Uncertainty on which patient groups to share study results with Authors reported that in some instances it was difficult to identify which patient groups to disseminate to (eg, studies conducted in emergency or acute settings).

Researcher-specific reasons for non-dissemination

Respondents spoke of unfamiliarity with the concept of disseminating results to patients and linked communities, it not being usual practice, not having received training in how to do it and perceiving that it was not their responsibility. Some were unsure of what and when they should disseminate, others felt it was unnecessary if participants had already been given their own clinical results or the trial reports were available in a journal publication or trial registry. Some indicated they were concerned about breaching copyright by sending journal articles to patients.

\section{Wider dissemination to patient groups}

Table 3 shows how respondents shared or planned to share results with patients beyond those who took part in the trial. Among the 1818 authors, 845 (46\%) shared or planned to share results with patients outside the trial via presentations at conferences with patient representation, $708(39 \%)$ by publishing lay summaries online, 577 $(32 \%)$ by direct contact with charities/patient groups, $494(27 \%)$ via mainstream media and 401 (22\%) via informal patient communities.

\section{Barriers and challenges to dissemination to patient groups}

Some felt disseminating to wider patient groups was easier than reaching trial participants, but others disagreed, citing lack of guidance on how to do this and not knowing how to find or approach relevant patient groups and organisations. Concern was also expressed about misinterpretation of results if the full context of the study was not understood-a particular risk, respondents suggested, if social media were used for dissemination. Mention was also made of raising expectations which can't be met if people were unable to access the intervention/treatment studied. Others noted that it was not possible to make clear recommendations on the basis of results from a single study. In addition, the dissemination of results from trials involving drugs sponsored by pharmaceutical companies was viewed as illegal off label promotion or advertising.

\section{External suggestions to disseminate}

Table 4 shows the sources of suggestions to disseminate to trial participants and wider patient groups. Relatively few respondents reported that dissemination was encouraged by institutional bodies such as research funders $(314,(17 \%)$ suggested dissemination to trial participants and $252(14 \%)$ suggested dissemination to other patient groups) or ethical review boards (333 (18\%) suggested dissemination to trial participants and 148 (8\%) suggested dissemination to other patient groups). Motivation to disseminate appears to have arisen more from within research teams rather than from institutional bodies.

\section{Patient involvement in dissemination}

A minority of the 1818 respondents reported they had, or planned to involve patients in dissemination activities (table 5): interpreting the study findings and potential impact of their study $(271,15 \%)$, developing the content of the materials for dissemination $(305,17 \%)$, contributing to writing or commenting on the manuscript (169, 9\%), selecting the most appropriate dissemination method $(295,16 \%)$, and presenting and sharing results with other patients or the public $(366,20 \%)$.

\section{DISCUSSION}

Our study shows that two-fifths of clinical trialists had disseminated results to trial participants (or planned to) up to 2 years after publishing their study. Half of those who had (or planned to) disseminate shared documents 
Table 4 Suggestions from relevant parties to disseminate results to trial participants and patient groups ( $\mathrm{n}=1818)$

\begin{tabular}{|c|c|c|c|}
\hline \multirow[b]{2}{*}{ Suggested dissemination } & \multicolumn{3}{|c|}{$\mathbf{N}(\%)$ of trials } \\
\hline & Yes & No & Don't know \\
\hline \multicolumn{4}{|l|}{ To trial participants } \\
\hline Other members of the research team & $708(39)$ & $598(33)$ & $217(12)$ \\
\hline $\begin{array}{l}\text { Patient and public involvement representative(s) involved } \\
\text { with the trial }\end{array}$ & $260(14)$ & $901(50)$ & $287(16)$ \\
\hline Ethical or institutional review board & $333(18)$ & $872(48)$ & $258(14)$ \\
\hline Academic institution & $302(17)$ & $880(48)$ & $259(14)$ \\
\hline Regulatory bodies & $144(8)$ & $931(51)$ & $320(18)$ \\
\hline \multicolumn{4}{|l|}{ To patient groups } \\
\hline Research funder(s) & $252(14)$ & $903(50)$ & $281(16)$ \\
\hline Ethical or institutional review board & $148(8)$ & $979(54)$ & $296(16)$ \\
\hline Academic institution & $238(13)$ & $917(50)$ & $284(16)$ \\
\hline Regulatory bodies & $83(5)$ & $971(53)$ & $339(19)$ \\
\hline
\end{tabular}

Percentages do not sum to $100 \%$ due to missing data.

prepared specifically for lay readers and a quarter shared both these and those written for an academic/clinical audience. Sharing with patients and patient groups more widely was mostly done via presentations at conferences where patients were represented or by publishing lay summaries on the trial website or a publicly accessible website. Sharing results directly with charitable organisations, and patient groups and communities, for example, via social media was less frequent. Furthermore, only a minority of authors involved patients in planning their dissemination strategies to study participants. Respondents reported that funders, academic institutions, ethical review boards and regulatory bodies rarely suggested dissemination to trial participants and linked patient groups. In addition, respondents described many perceived barriers and practical challenges to dissemination.

Previous studies describing the frequency of sharing study results with participants have taken different approaches, and heterogeneity makes direct comparisons

Table 5 Patient involvement in dissemination of the research

\begin{tabular}{|c|c|c|c|c|}
\hline \multirow[b]{2}{*}{ Type of patient involvement } & \multicolumn{4}{|l|}{$\mathbf{N}(\%)$ of trials } \\
\hline & $\begin{array}{l}\text { Already asked for this } \\
\text { involvement }\end{array}$ & $\begin{array}{l}\text { Plan to ask for this } \\
\text { involvement }\end{array}$ & $\begin{array}{l}\text { No plan to ask for } \\
\text { this involvement }\end{array}$ & Don't know \\
\hline $\begin{array}{l}\text { Developing the content of the } \\
\text { materials for dissemination }\end{array}$ & $178(10)$ & $127(7)$ & $1105(61)$ & $151(8)$ \\
\hline $\begin{array}{l}\text { Selecting most appropriate } \\
\text { dissemination method }\end{array}$ & $155(9)$ & $140(8)$ & $1072(59)$ & $178(10)$ \\
\hline $\begin{array}{l}\text { Presenting/sharing results with } \\
\text { other patients or the public }\end{array}$ & $211(12)$ & $155(9)$ & $998(55)$ & $187(10)$ \\
\hline
\end{tabular}

Percentages do not sum to $100 \%$ due to missing data. 
difficult. ${ }^{6}{ }^{17-20}$ Recent research includes a survey of over 3000 health research participants in the USA with $33 \%$ reporting they had received results from a study they had participated in ${ }^{19}$ and a survey of 414 researchers in the USA with $56 \%$ able to recall studies for which they were investigators where results were not disseminated to participants. ${ }^{20}$ A survey of authors of community participation studies showed dissemination to participants and the public occurred in $98 \%$ of studies but few included details about it in publications. ${ }^{18}$

Respondents to our survey indicated that few research funders, regulators, ethical review boards, and academic institutions requested dissemination to participants. And while we found a higher proportion of requests from ethical review boards (18\%) than an earlier study in the USA $(7 \%),{ }^{17}$ this is still low. Recent reviews of informed consent documentation at Canadian research institutions $^{23}$ and policies and recommendations for dissemination by major US research funders ${ }^{24}$ have also revealed a lack of support for disseminating results to patients. Our finding that only a minority of authors involved patients in their dissemination plans is very much in line with a previous survey. ${ }^{25}$ Similarly, our respondents' reports of barriers to dissemination have been reported in previous smaller surveys and include: concern about emotional effects on participants; participants' difficulty understanding results; logistical issues; and researcher, financial and regulatory barriers. ${ }^{6202126}$ The potential for dissemination to participants and patient communities being harmful should not be overlooked. Findings from scientific studies are not always reported objectively and their clinical importance can be amplified and distorted via press offices, mainstream media outlets and social media. Misunderstanding of the clinical importance of study results may lead to unintended consequences including provoking unjustified concern or false hope among patients, which may result in increased clinical visits and requests for inappropriate treatment. Poor reporting of study results poses a problem for both health professionals and the patient community. Rigorous accurate, accessible, and objective reporting is in everyone's interest.

\section{Study strengths and limitations}

This is the largest cross-disciplinary survey to date about the dissemination of results of clinical trials to patients. Asking respondents about practice in relation to specific studies rather than for general opinions and practice provides a better sense of the frequency of dissemination. However, our study has several limitations. While the response rate is similar to other surveys conducted with researchers ${ }^{27}$ and we generated a large and varied sample, the generalisability of the results may be compromised by the low response rate. We do not know if responders differed from non-responders in terms of the characteristics of the trials, the authors, or the funding received; it is possible that those who responded were more likely to have disseminated than those who did not.
One reason for the low response rate may be our general search of PubMed to identify clinical trials, because the number of authors who informed us their study was not a clinical trial (eg, it was a protocol or methodological paper) suggests that many may have ignored the invitation. Furthermore, some will not have received it, with approximately $18 \%$ of email addresses of authors in PubMed estimated to be invalid. ${ }^{29}$ We also restricted the sample to journals in English. Additionally, by using Perl script to extract author details we were not able to include reports of trials where there was no email address in the relevant field, where the email address was not in the format abcd@xyz or where moderators prevented automatic extraction by substituting the @ symbol. Finally, we did not exclude published protocols from the sample so the proportion of authors who had already disseminated would probably have been higher if we had.

\section{Study implications}

The call for dissemination of trial results to participants is not new but our work shows that it is still not regarded as an essential component of the research process. Many respondents had not considered it and only half asked participants if they wanted to be informed of the study results. This is surprising given that The Declaration of Helsinki requires this ${ }^{9}$ and respondents indicated that dissemination to participants and patient communities has the potential to improve public trust in medical research, encourage people to participate, raise health literacy and increase uptake of research findings.

Dissemination of results to patients is a shared responsibility of funders, regulators, ethical review boards, universities and researchers. Yet our study makes it clear that that the latter get little guidance and support for it from the former. We clearly need a better understanding of why this is and what can be done to support and motivate researchers to share results and make dissemination an integral part of the research process. Our findings suggest the following:

1. Clear policy and practical guidance.

Explicit policy and clear guidance are needed on when, what, and how to share results with patients and their linked communities. This should be developed and informed by the best available evidence, which needs to come from research testing different formats and modes of delivery.

2. Incentivise dissemination by making it mandatory.

Funding organisations should incentivise dissemination beyond publication in peer reviewed journals. ${ }^{14}$ They could (as a few funders currently do) make research funding conditional on the inclusion of appropriate plans for dissemination to participants and linked communities in accessible formats. ${ }^{1124}$ In addition they could allocate money and support for these activities, offer training to improve grantees' competency in this respect, ${ }^{24}$ make the expectations of researchers clearer, ${ }^{30}$ and support research to identify effective dissemination strategies. While some organisations are 
already striving to facilitate dissemination to patients, even where this practice is encouraged, more could and should be done to equip researchers with the necessary skills and resources.

3. Increased scrutiny from ethical review boards.

Ethical review boards should routinely review the inclusion and appropriateness of investigators' dissemination plans to participants ${ }^{11} 12$ and linked communities. With the sometimes-lengthy delay between study recruitment and study publication, researchers need a clear steer from the outset about when and how to contact participants and linked organisations. ${ }^{8}$ They also need guidance on how to disseminate results that have the potential to cause harm to patients and unnecessary burden on health professionals if misunderstood.

4. Encourage early planning of dissemination.

Researchers should plan for dissemination in the early phases of their work and include these plans in their study protocols, informed consent procedures and applications for ethical approval. ${ }^{11} 1223$ Good practice could be to ask study participants (and/or next of kin) when seeking consent if they want to receive results (as interest in receiving results varies ${ }^{153132}$ ) and to inform them that they will be asked again at the end of the study. Re-offering results in this way moves the onus away from patients having to request them while respecting their choice and right to decline. ${ }^{57}$

5. Improve training.

Researchers receive little training in communicating findings to stakeholders, and most spend less than $10 \%$ of their time on all dissemination activities. ${ }^{25}$ They need better guidance on how to plan, resource and implement dissemination, ${ }^{2}$ particularly to patients and their linked communities. Universities should teach that dissemination to patients and the public is a core element of good practice and equip researchers with the skills and support to communicate effectively with patients, the public and media outlets. ${ }^{14}$ Investigators should be taught not to make assumptions about whether and what results patients may understand and value. ${ }^{11}$ Increasingly networked patient communities are adept and skilled at accessing and communicating the results of research to members. And the patient community as a whole is interested in both positive and negative findings. ${ }^{12}$ Researchers need to better understand that objective unsensational reporting is crucial for health professionals, policy makers, patients and the public. They need to consider the potential for results to cause unjustified concern and potential harm if they are over interpreted and spun in a way which exaggerates their importance. All end users of research, whether they are clinicians, policy makers or patients, need to understand the limitations of a single study in the context of existing knowledge.

6. Development of standards.

Academic institutions should develop standardised content for patient information leaflets about participating in clinical trials and in the patient consent forms. This should include details of when, how and what results will be shared to ensure there is transparency about this and help promote the importance of dissemination to participants. ${ }^{23}$ Standards for the content of dissemination materials for participants and linked communities should be developed and adopted for all types of health research. To avoid misinformation and the potential for harm, these should include sections on how to interpret the results, study limitations and implications for management and treatment (as applicable).

7. Support from journals.

Journals have a key role in dissemination and translation of knowledge into practice. Currently, patients and the public have limited access to the results of research, as much of the scientific literature still requires a subscription. ${ }^{33}$ Even if access is possible, articles may not be understandable to generalists and lay audiences for most are written for narrow specialist clinical and academic audiences. Journal editors should promote open access research and support its dissemination in a form which can readily be understood and encourage this by asking researchers to report how they have done this or plan to do so. ${ }^{34}$ Journals should also encourage authors to write clear abstracts which are accessible to a non-specialist general medical audience, and informed patient communities and in addition consider publishing lay summaries, and upload these as open access supplementary files. Journals publishing protocols can ask authors to describe how their research will be shared with participants, to encourage early planning.

Acknowledgements Rosamund Snow, former Patient editor at The BMJ, died before this work was completed, but was key to instigating this research and championing the rights of patients to have access to study results. We thank Sally Crowe and Paul Wickes, members of The BM ${ }^{\prime}$ s international patient advisory panel, for their comments on the survey and draft paper; and the volunteers who pilot tested the survey to help us improve the survey questions. We will share the results of the survey, via a link to the publication of this paper, with all participants who indicated that they wished to receive the results. We will disseminate through social media and by targeting patient communities; we will also share with all The BMJ's patient and public reviewers and ask them to share within their own networks. We will share the paper with journal editors to encourage them to get authors to think about dissemination to participants and patient groups.

Contributors SS and AP wrote the study protocol. SS administered the surveys, conducted the data analysis and wrote the first draft of the manuscript. MM conducted the PubMed searches. All authors (SS, AP, TR, MM and MC) developed the survey questions, commented on the results, helped write the paper for publication, approved the final version of the paper and agreed on the dissemination plan.

Funding MM was partially funded by Croatian Science Foundation, grant No. IP-2014-09-7672 Professionalism in Health Care.

Competing interests SS and TR are employed by The BMJ which has a patient partnership initiative. AP works on a freelance basis for The BMJ and is a senior research advisor at Stanford University, where she is responsible for advising and seeking funding on dissemination of research studies. AP and TR are patients with long-term medical conditions and are committed to increasing the dissemination of results to patients. MC has been involved in many clinical trials and systematic reviews, such that he has had to organise the dissemination of his own research and use material disseminated by others. He seeks funding for these trials and reviews, as well as for research into methodology, including dissemination. 
Patient consent for publication Not required.

Ethics approval University of Oxford Central University Research Ethics Committee (MS-IDREC-C1-2013-174)

Provenance and peer review Not commissioned; externally peer reviewed.

\section{Data availability statement}

Deidentified participant data are available from the corresponding author ( sschroter@bmj.com) on reasonable request.

Open access This is an open access article distributed in accordance with the Creative Commons Attribution Non Commercial (CC BY-NC 4.0) license, which permits others to distribute, remix, adapt, build upon this work non-commercially, and license their derivative works on different terms, provided the original work is properly cited, appropriate credit is given, any changes made indicated, and the use is non-commercial. See: http://creativecommons.org/licenses/by-nc/4.0/.

\section{ORCID iDs}

Sara Schroter http://orcid.org/0000-0002-8791-8564

Amy Price http://orcid.org/0000-0002-5200-7322

Mike Clarke http://orcid.org/0000-0002-2926-7257

\section{REFERENCES}

1 Tetroe JM, Graham ID, Foy R, et al. Health research funding agencies' support and promotion of knowledge translation: an international study. Milbank Q 2008;86:125-55.

2 Wilson PM, Petticrew M, Calnan MW, et al. Does dissemination extend beyond publication: a survey of a cross section of public funded research in the UK. Implementation Sci 2010;5.

3 Scherer RW, Meerpohl JJ, Pfeifer N, et al. Full publication of results initially presented in Abstracts. Cochrane Database Syst Rev 2018;11.

4 Rawat S, Meena S. Publish or perish: where are we heading? J Res Med Sci 2014;19:87-9.

5 Fernandez CV, Kodish E, Weijer C. Informing study participants of research results: an ethical imperative. IRB 2003;25:12-19.

6 Partridge $\mathrm{AH}$, Hackett $\mathrm{N}$, Blood $\mathrm{E}$, et al. Oncology physician and nurse practices and attitudes regarding offering clinical trial results to study participants. J Natl Cancer Inst 2004;96:629-32.

7 Shalowitz DI, Miller FG. The search for clarity in communicating research results to study participants. J Med Ethics 2008;34:e17.

8 Lévesque E, Joly Y, Simard J. Return of research results: general principles and international perspectives. J Law Med Ethics 2011;39:583-92.

9 World Medical Association. World Medical association Declaration of Helsinki: ethical principles for medical research involving human subjects. JAMA 2013;310:2191-4.

10 European Medicines Agency. Posting of clinical trial summary results in European clinical trials database (EudraCT) to become mandatory for sponsors as of 21 July 2014, 2014. Available: http://www.ema. europa.eu/ema/index.jsp?curl=pages/news_and_events/news/2014/ 06/news_detail_002127.jsp\&mid=WC0b01ac058004d5c1 [Accessed 29 May 2019].

11 National Academies of Sciences, Engineering, and Medicine. Returning individual research results to participants: guidance for a new research paradigm. Washington, DC: The National Academies Press, 2018.

12 Shalowitz DI, Miller FG. Communicating the results of clinical research to participants: attitudes, practices, and future directions. PLoS Med 2008;5:e91.
13 Clayton EW, Ross LF. Implications of disclosing individual results of clinical research. JAMA 2006;295:37-8.

14 Waddell C. So much research evidence, so little dissemination and uptake: mixing the useful with the pleasing. Evid Based Ment Health 2001;4:3-5.

15 Dixon-Woods M, Jackson C, Windridge KC, et al. Receiving a summary of the results of a trial: qualitative study of participants views. BMJ 2006;332:206-10.

16 The Lancet. Trialists should tell participants results, but how? The Lancet 2006;367:1030.

17 Rigby H, Fernandez CV. Providing research results to study participants: support versus practice of researchers presenting at the American Society of hematology annual meeting. Blood 2005;106:1199-202.

18 Chen PG, Diaz N, Lucas G, et al. Dissemination of results in community-based participatory research. Am J Prev Med 2010;39:372-8.

19 Long CR, Stewart MK, Cunningham TV, et al. Health research participants' preferences for receiving research results. Clin Trials 2016;13:582-91.

20 Long CR, Purvis RS, Flood-Grady E, et al. Health researchers' experiences, perceptions and barriers related to sharing study results with participants. Health Res Policy Syst 2019;17.

21 Cox K, Moghaddam N, Bird L, et al. Feedback of trial results to participants: a survey of clinicians' and patients' attitudes and experiences. Eur J Oncol Nurs 2011;15:124-9.

22 Purvis RS, Abraham TH, Long CR, et al. Qualitative study of participants' perceptions and preferences regarding research dissemination. AJOB Empirical Bioethics 2017;8:69-74.

23 Curran D, Kekewich M, Foreman T. Examining the use of consent forms to promote dissemination of research results to participants. Res Ethics 2019:15:1-28.

24 McElfish PA, Purvis RS, Stewart MK, et al. Health research funding agencies' policies, recommendations, and tools for dissemination. Prog Community Health Partnersh 2018;12:473-82.

25 Brownson RC, Jacobs JA, Tabak RG, et al. Designing for dissemination among public health researchers: findings from a national survey in the United States. Am J Public Health 2013;103:1693-9.

26 Fernandez CV, Kodish E, Shurin S, et al. Offering to return results to research participants: attitudes and needs of principal Investigators in the children's Oncology Group. J Pediatr Hematol Oncol 2003;25:704-8.

27 Mulligan A, Hall L, Raphael E. Peer review in a changing world: an international study measuring the attitudes of researchers. J Am Soc Inf Sci Tec 2013;64:132-61

28 Price A, Schroter S, Clarke M, et al. Role of supplementary material in biomedical Journal articles: surveys of authors, reviewers and readers. BMJ Open 2018;8:e021753.

29 Rodriguez-Esteban R, Vishnyakova D, Rinaldi F. Revisiting the decay of scientific email addresses. bioRxiv.

30 Wilson PM, Petticrew M, Calnan MW, et al. Disseminating research findings: what should researchers do? A systematic scoping review of conceptual frameworks. Implementation Sci 2010;5.

31 Partridge $\mathrm{AH}$, Burstein HJ, Gelman RS, et al. Do patients participating in clinical trials want to know study results? J Natl Cancer Inst 2003;95:491-2.

32 Partridge $\mathrm{AH}$, Wong JS, Knudsen $\mathrm{K}$, et al. Offering participants results of a clinical trial: sharing results of a negative study. Lancet 2005;365:963-4.

33 Piwowar $\mathrm{H}$, Priem J, Larivière $\mathrm{V}$, et al. The state of $\mathrm{OA}$ : a large-scale analysis of the prevalence and impact of open access articles. PeerJ 2018;6:e4375.

34 Wicks P, Richards T, Denegri S, et al. Patients' roles and rights in research. BMJ 2018;362. 